\title{
STABLE RANK OF SUBALGEBRAS OF THE DISC ALGEBRA
}

\author{
RUDOLF RUPP
}

(Communicated by Paul S. Muhly)

\begin{abstract}
We calculate the stable rank of subalgebras (in the algebraic sense) of the disc algebra. We extend the results to subalgebras of $A(\bar{G})$, where $G$ is a bounded domain whose boundary consists of finitely many closed nonintersecting Jordan curves.
\end{abstract}

\section{INTRODUCTION}

The concept of the stable rank of a ring, introduced, by $\mathrm{H}$. Bass [1], has been very useful in treating some problems in algebraic $K$-theory. In a serial of papers G. Corach and F. D. Suárez calculated the stable rank of Banach algebras of holomorphic functions. Among them are the well-known algebras $A(K)$ of all continuous complex valued functions on a compact set $K \subset \mathbf{C}$ which are analytic in its interior. However, the stable rank of the disc algebra $A(\overline{\mathbf{D}})$ was first calculated by P. Jones, D. Marshall and T. Wolff [8] by an entirely different technique.

In this paper we study the stable rank of subalgebras of the disc algebra $A(\overline{\mathbf{D}})$. Generalizations to subalgebras of $A(\bar{G})$ are given, where $G$ denotes a bounded domain whose boundary consists of finitely many nonintersecting closed Jordan curves. However, the best results are obtained for subalgebras of the disc algebra $A(\overline{\mathbf{D}})$.

Since our algebras need not be normable, our results are generalizations of results of G. Corach and F. D. Suárez, see [3]. Also this paper complements the results in [10].

1. It is well known that the group of units in Banach algebras is open. Unfortunately, this feature is lost in the general case of a topological algebra. This led to the following definitions:

A topological algebra $A$ is called a $Q$-algebra if the set of units, $A^{x}$, is open in $A$.

In this paper we consider complex, commutative $Q$-algebras with unit element being denoted by 1 .

Received by the editors February 10, 1989.

1980 Mathematics Subject Classification (1985 Revision). Primary 46J15; Secondary 18 F25. 
Given a $Q$-algebra $A$, an element $a \in A^{n}$ is called unimodular if there exists $b \in A^{n}$ such that

$$
\langle b, a\rangle:=\sum_{i=1}^{n} b_{i} a_{i}=1 .
$$

We denote by $U_{n}(A)$ the set of unimodular elements of $A^{n}$. Finally, $a=$ $\left(a_{1}, \ldots, a_{n}\right) \in U_{n}(A)$ is called reducible if there exists $x_{1}, \ldots, x_{n-1}$ in $A$ such that

$$
\left(a_{1}+x_{1} a_{n}, \ldots, a_{n-1}+x_{n-1} a_{n}\right) \in U_{n-1}(A) .
$$

The stable rank of $A$, denoted by $\operatorname{sr}(A)$, is the least integer $n$ such that every $a \in U_{n+1}(A)$ is reducible.

From the theory of the stable rank of $Q$-algebras we mention the following fact; see [9, p. 18, Korollar 1] or [4, Proposition 1]. (The proof given there is also valid in $Q$-algebras.)

Proposition 1.1. Suppose that $A$ is a Q-algebra, $\gamma:[0,1] \rightarrow \mathbf{C}$ is a continuous curve and let $\Gamma:[0,1] \rightarrow U_{2}(A), \Gamma(t):=(a-\gamma(t), b)$ such that $(a-\gamma(0), b)$ is reducible. Then $(a-\gamma(1), b)$ is reducible, too.

2. Let $K$ be a compact set of the plane $\mathrm{C}$ and let $A(K)$ denote the Banach algebra of all complex valued continuous functions which are holomorphic in the interior $K^{0}$ of $K$.

Let $A$ be a subalgebra of $A(K)$ in the algebraic sense. We say that the Nullstellensatz holds in $A$ iff the following condition holds for every $n:\left(f_{1}, \ldots\right.$, $f_{n}$ ) is unimodular iff the functions $f_{1}, \ldots, f_{n}$ have no common zero in $K$. If the last condition is known to hold for $n=1,2$, we say that the weak Nullstellensatz holds in $A$. If, finally, this condition is known to be true for $n=1$ then $A$ is said to be inversionally closed. For example, the Nullstellensatz holds in the well-known disc algebra $A(\overline{\mathbf{D}})$.

Suppose now that the weak Nullstellensatz holds in the algebra $A \subset A(K)$ The inversion set $I(f, g)$ of $f, g \in A$ is defined to be the set of all complex numbers $a$ such that the vector $(f-a, g)$ is unimodular, for short,

$$
I(f, g)=\left\{a \in \mathbf{C}:(f-a, g) \in U_{2}(A)\right\} .
$$

Since the weak Nullstellensatz holds in $A$, we can restate this as follows:

$$
\mathrm{C} \backslash I(f, g)=f\left(Z_{g}\right),
$$

where $Z_{g}$ denotes the set of zeros of $g$ in $K$. This identity shows that every number $\lambda \in \mathbf{C} \backslash f(K)$ is a member of the inversion set of $I(f, g)$, moreover, $(f-\lambda, g)$ is reducible, since the algebra $A$ is inversionally closed.

Remarks. If the weak Nullstellensatz holds in $A \subset A(K)$, then the algebra $A$, endowed with the topology of uniform convergence, is a $Q$-algebra.

The author has no example of an algebra $A$ for which the weak Nullstellensatz holds, but the Nullstellensatz does not hold.

In view of Proposition 1.1 the inversion set $I(f, g)$ plays an important role. 
In this paragraph, we will consider mainly subalgebras of the algebra $H^{1,1}(\overline{\mathbf{D}})$, where $H^{1,1}(\overline{\mathbf{D}})$ denotes all those functions of the disc algebra $A(\overline{\mathbf{D}})$ whose boundary function is absolutely continuous, i.e.

$$
H^{1,1}(\overline{\mathbf{D}}):=\left\{f \in A(\overline{\mathbf{D}}):\left.f\right|_{\partial \mathbf{D}} \text { is absolutely continuous }\right\} .
$$

Further information on $H^{1,1}(\overline{\mathbf{D}})$ is available in [5], Theorem 3.10.

Theorem 2.1. let $A$ be a subalgebra of $H^{1,1}(\overline{\mathbf{D}})$ in which the weak Nullstellensatz holds, and suppose that $(f, g)$ is unimodular, where $g$ is not the zero function. Then the complement $\mathbf{C} \backslash I(f, g)$ of the inversion set is totally disconnected.

Proof. Suppose on the contrary that there exists a closed connected subset $J$ of the compact set $\mathbf{C} \backslash I(f, g)$ which contains more than one point. Since the weak Nullstellensatz holds in $A$, we have

$$
\mathrm{C} \backslash I(f, g)=f\left(Z_{g}\right) .
$$

Moreover, since $J$ does not have isolated points, for every given point $w \in J$ there exists a sequence $\left(z_{n}\right)_{n \in \mathbf{N}}$ such that

$$
\lim _{n \rightarrow \infty} f\left(z_{n}\right)=w, \quad g\left(z_{n}\right)=0 \quad(n \in \mathbf{N}) .
$$

The identity theorem for analytic functions implies that every accumulation point of $\left(z_{n}\right)_{n \in \mathbf{N}}$ has unit modulus. So there exists a compact set $E \subset \partial \mathbf{D}$ such that

$$
J=f(E), \quad g(E)=\{0\} .
$$

As a subset of a set of Lebesgue measure zero, $E$ also has Lebesgue measure zero.

Let $p_{x}$ resp. $p_{y}$ denote the projection on the real resp. imaginary axis.

Since both projections are continuous and $J$ is connected, the sets $p_{x}(J)$ and $p_{y}(J)$ are intervals on the real resp. imaginary axis. On the other hand, they are the absolutely continuous image of the set $E \subset \partial D$ which has Lebesgue measure zero. So they also have Lebesgue measure zero and consist therefore of exactly one point. Thus we have the contradiction that $J$ consists of one point.

This theorem is the main ingredient in proving the following result.

Theorem 2.2. Let $A$ be a subalgebra of $H^{1,1}(\overline{\mathbf{D}})$ in which the weak Nullstellensatz holds. Then its stable rank is one.

Remarks. It should be noted that no assumptions on topology for the algebra $A$ are made.

To prove Theorem 2.2 we use the fact that $f\left(Z_{g}\right)$ is totally disconnected. This argument has been used before, implicitly or explicitly, see [2, Theorem 1.13 ] or [4, Corollary 2].

Proof. We have to show that every unimodular vector $(f, g)$ is reducible. Since the weak Nullstellensatz holds in $A$, this is equivalent to the existence of a 
function $h \in A$ such that $f+h g$ is zerofree in $\overline{\mathbf{D}}$. Of course, we can assume that $g$ is not the zero function. By Proposition 1.1 and the subsequent remark it is enough to exhibit a path $\gamma$ such that $(f-\gamma(t), g)$ is unimodular, $\gamma(0)=0$ and $(f-\gamma(1), g)$ is reducible. By definition $(f-a, g)$ is unimodular iff $a \in I(f, g)$. Theorem 2.1 implies that $\mathbf{C} \backslash I(f, g)$ is totally disconnected and compact, that is, its (covering) dimension is zero ([7], p. 20, Section A). By a result of plane topology, see [7], Theorem IV.4, the open set $I(f, g)$ is connected (and unbounded). Choose any point $b \notin f(\overline{\mathbf{D}})$ and a path $\gamma \subset$ $I(f, g)$ joining 0 and $b$. Since $A$ is inversionally closed we have $b \in I(f, g)$. An inspection shows that $\gamma$ has the required properties.

This theorem implies that the following algebras have stable rank one:

$$
\begin{aligned}
A^{N}(\overline{\mathbf{D}}) & :=\left\{f \in A(\overline{\mathbf{D}}): f^{(n)} \in A(\overline{\mathbf{D}}), n=1, \ldots, N\right\} ; \\
A^{\infty}(\overline{\mathbf{D}}) & :=\left\{f \in A(\overline{\mathbf{D}}): f^{(n)} \in A(\overline{\mathbf{D}}) \text { for all numbers } n\right\} .
\end{aligned}
$$

Note that the topological algebra $A^{\infty}(\overline{\mathbf{D}})$ is not normable, [11].

3. It is natural to ask whether Theorem 2.1 holds in general domains.

Theorem 3.1. Let $G$ be a bounded domain whose boundary consists of finitely many nonintersecting closed Jordan curves. Assume further that $f$ is holomorphic in $\bar{G}$ and $g \in A(\bar{G})$ is not identical zero. Then the compact set $f\left(Z_{g}\right)$ is totally disconnected.

Proof. Suppose on the contrary that there exists a continuum $J$ in $f\left(Z_{g}\right)$. Since $J$ cannot contain isolated points, the identity theorem for holomorphic functions implies the existence of a compact set $K \subset \partial G$ such that

$$
J=f(K) \text { and } g(K)=\{0\} .
$$

It is well known from topology that there exist continua $J^{\prime} \subset J$ with diameter less than a given positive number $\varepsilon$. Since $f^{\prime}$ is also holomorphic in $\bar{G}$, we can therefore assume that no point of $J$ is a critical point of $f$, that is, we have

$$
J=f(K), \quad g(K)=\{0\} \quad \text { and } 0 \notin f^{\prime}(K) .
$$

Now the function $f$ is locally injective on $K$, and a standard argument shows the existence of compact sets $K_{j}, j=1, \ldots, n$ such that

$$
K=\bigcup_{j=1}^{n} K_{j} \quad \text { and }\left.f\right|_{K_{j}} \text { is injective. }
$$

This yields

$$
J=f(K)=\bigcup_{j=1}^{n} f\left(K_{j}\right) .
$$

Now the set $Z_{g} \cap \partial G$ is totally disconnected. (A continuum would be on exactly one Jordan curve $\Gamma$, so it is an arc. The well-known two-constant theorem (see [6], p. 299, Satz 2) implies the contradiction $g=0$. 
Every compact subset $K_{j}$ of $Z_{g} \cap \partial G$ is also totally disconnected. Their (covering) dimension is therefore zero ([7], p. 20, Section A). Since the functions $\left.f\right|_{K_{j}}$ are homeomorphisms, the compact sets $f\left(K_{j}\right)$ also have dimension zero.

By the sum theorem in dimension theory $J$ has dimension zero, too ([7], Theorem III.2). Because $J$ is compact, this implies that $J$ is totally disconnected. But by assumption $J$ was a continuum, which is a contradiction. Therefore $f\left(Z_{g}\right)$ is totally disconnected.

We will use this result to calculate the stable rank of some algebras.

Let $K$ be a compact plane set. We say that a subalgebra $A$ of $A(K)$ is a holomorphically generated $Q$-algebra, for short, $H G Q$-algebra iff the following two conditions hold:

(i) $A$ is a $Q$-algebra.

(ii) There exists a set $L$ of functions holomorphic on $K$ such that $1 \in L$, and in each neighborhood of a suitable element of $A$ we find a member of $L$, that is the closure of $L$ is $A$.

For example, the disc algebra $A(\overline{\mathbf{D}})$ is a $H G Q$-algebra.

Theorem 3.2. Let $G$ be a bounded domain whose boundary consists of finitely many closed, nonintersecting Jordan curves. Suppose $A \subset A(\bar{G})$ is a $H G Q-$ algebra, in which the weak Nullstellensatz holds. Then its stable rank is one.

Proof. We have to show that every unimodular vector $(f, g) \in A^{2}$ is reducible. As before, we may assume that $g \neq 0$.

Step 1. $f$ is holomorphic in $\bar{G}$. We argue as in the proof of Theorem 2.2. We have to replace the use of Theorem 2.1 by the use of Theorem 3.1.

Step 2. $f \in A$. Since the vector $(f, g)$ is unimodular, there exist $\alpha, \beta \in A$ such that

$$
\alpha f+\beta g=1 .
$$

Because $A$ is a $H G Q$-algebra, we can approximate the function $\alpha$ by functions which are holomorphic in $\bar{G}$. Especially $A$ is a $Q$-algebra, so there exists a neighborhood $U$ of 1 such that every member of $U$ is invertible in $A$. Now multiplication by $f$ is a continuous operation on $A^{2}$, so there exist functions $\tilde{\alpha} \in A$ holomorphic in $\bar{G}$ such that $\tilde{\alpha} f+\beta g=: u$ is invertible in $A$. This identity implies that the vector $(\tilde{\alpha}, g)$ is unimodular, hence reducible by the first step. So we have $h \in A, v \in A^{x}$ such that

$$
\tilde{\alpha}+u g=v .
$$

Now we insert this in the equation $\tilde{\alpha} f+\beta g=u$ and are done.

We will give two examples.

$$
\begin{aligned}
\Lambda_{c(}(\overline{\mathbf{D}}):=\{f \in A(\overline{\mathbf{D}}): & f \text { satisfies a Hölder-Lipschitz condi- } \\
& \text { tion on } \overline{\mathbf{D}} \text { of order } \alpha\}
\end{aligned}
$$


and

$$
A_{0}^{\infty}(\overline{\mathbf{D}}):=\left\{f \in A^{\infty}(\overline{\mathbf{D}}): f^{\prime}(0)=0\right\} .
$$

The assumptions for Theorem 3.2 are easily verified, so the stable rank of both algebras is one.

\section{ACKNOWLEDGMENT}

I want to thank Professor N. Steinmetz for valuable comments during the preparation of this paper.

\section{REFERENCES}

1. H. Bass, $K$-theory and stable algebra, Inst. Hautes Etudes Sci. Publ. Math. 22 (1964), 5-60.

2. G. Corach and F. D. Suárez, Extension problems and stable rank in commutative Banach algebras, Topology Appl. 21 (1985), 1-8.

3. __ Stable rank in holomorphic function algebras, Illinois J. Math. 29 (1985), 627-639.

4. $\ldots$, On the stable rank of uniform algebras and $H^{\infty}$, Proc. Amer. Math. Soc. 98 (1986), 607-610.

5. P. L. Duren, Theory of $H^{p}$-spaces, Academic Press, New York and London, 1970.

6. G. M. Golusin, Geometrische Funktionentheorie, VEB Deutscher Verlag der Wissenschaften, Berlin, 1957.

7. W. Hurewicz and H. W. Wallman, Dimension theory, Princeton Univ. Press, 1941.

8. P. W. Jones, D. Marshall and T. Wolff, Stable rank of the disc algebra, Proc. Amer. Math. Soc. 96 (1986), 603-604.

9. R. Rupp, Über den Bass-Stable-Rank komplexer Funktionen-Algebren, Dissertation, Karlsruhe, 1988.

10. __ Stable rank of holomorphic function algebras (preprint).

11. W. P. Novinger, Holomorphic functions with infinitely differentiable boundary values, Illinois J. Math. 15 (1971), 80-90.

Universität KarlsRuhe, Mathematisches Institute I, ENGLERStr. 2, D-7500 KarlsRUhe 1 